Annuaire du Collège de France 2017-2018

\title{
Civilisation mésopotamienne
}

\section{Dominique Charpin}

\section{OpenEdition}

\section{Journals}

Édition électronique

URL : https://journals.openedition.org/annuaire-cdf/15574

DOI : 10.4000/annuaire-cdf.15574

ISBN : 978-2-7226-0572-5

ISSN : 2109-9227

Éditeur

Collège de France

Édition imprimée

Date de publication : 30 décembre 2020

Pagination : 187-208

ISBN : 978-2-7226-0516-9

ISSN : 0069-5580

Référence électronique

Dominique Charpin, «Civilisation mésopotamienne », L'annuaire du Collège de France [En ligne], 118| 2020, mis en ligne le 31 mars 2021, consulté le 22 août 2022. URL : http://journals.openedition.org/ annuaire-cdf/15574 ; DOI : https://doi.org/10.4000/annuaire-cdf.15574 


\title{
CIVILISATION MÉSOPOTAMIENNE
}

\author{
Dominique CHARPIN
}

Membre correspondant de l'Académie des inscriptions et belles-lettres, professeur au Collège de France

Mots-clés : Mésopotamie, Ur, Babylone, Samsu-iluna

La série de cours "La ville d'Ur à l'époque paléo-babylonienne » est disponible en audio et en vidéo, sur le site internet du Collège de France (https://www.college-defrance.fr/site/dominique-charpin/course-2017-2018.htm), ainsi que les conférences d'Elisabeth Stone (http://www.college-de-france.fr/site/dominique-charpin/guestlecturer2017-2018_1.htm) et de Paul Zimansky (http://www.college-de-france.fr/site/ dominique-charpin/guestlecturer-2017-2018.htm).

\section{ENSEIGNEMENT}

COURS - LA VILLE D'UR À L'ÉPOQUE PALÉO-BABYLONIENNE ${ }^{1}$

\section{Introduction}

Le choix d'étudier la ville d'Ur à l'époque paléo-babylonienne fait suite à une actualité à la fois scientifique et personnelle : la reprise de la fouille de ce site du Sud irakien sous la direction d'Elisabeth Stone lors de deux campagnes à l'automne 2015 et au printemps 2017, auxquelles Dominique Charpin a participé en tant qu'épigraphiste. Le cours (et le séminaire) s'intégraient par ailleurs au projet «EcritUr » proposé par D. Charpin à l'ANR et pour lequel un financement a été obtenu pour trente-six mois à partir du 1 ${ }^{\mathrm{er}}$ octobre 2017.

1. Les mots sumériens sont rendus en minuscules (gudu 4 dub-lá-mah), les mots akkadiens en italiques (entum), les idéogrammes dans les textes akkadiens en capitales romaines (A.AB.BA). 


\title{
Cours 1 - Histoire des fouilles et des publications
}

\author{
4 avril 2018
}

Le site d'Ur se reconnaît de loin en raison de l'imposante masse de sa tour à étages, autrement dit sa ziggourat ; le nom arabe moderne du site, Tell al-Muqayyar, signifie la « colline du bitume », car ce matériau a été utilisé comme mortier dans la construction de cet édifice.

Pietro della Valle est le premier explorateur occidental qui ait mentionné le site d'Ur, dans la seconde moitié du XVII ${ }^{\mathrm{e}}$ siècle. Les premières fouilles sont dues à John G. Taylor, vice-consul britannique à Basra, de 1853 à 1854. Il découvrit des cylindres d'argile inscrits ainsi que des briques portant l'empreinte d'inscriptions qui permirent à Henry Rawlinson en 1862 d'identifier Tell al-Muqayyar comme la ville d'Ur, qu'on rapprocha aussitôt d' «Ur Kasdim », patrie d'Abraham selon la Genèse. Taylor découvrit aussi une trentaine de tablettes qui furent envoyées au British Museum, où elles furent mélangées avec celles issues des fouilles que Loftus avait menées au même moment à Tell Sifr ; c'est seulement en 1980 qu'elles ont été correctement identifiées ${ }^{2}$. Des fouilles non documentées furent pratiquées dans les décennies suivantes, heureusement à une échelle relativement limitée : environ deux cents tablettes paléo-babyloniennes d'Ur se trouvent de ce fait réparties dans diverses collections. Juste après la Première Guerre mondiale, l'exploration du site reprit, avec Reginald Campbell Thompson en 1918, puis Harry Reginald Holland Hall en 1919.

Un mandat sur l'Irak nouvellement créé à partir des dépouilles de l'Empire ottoman fut alors confié par la Société des Nations à la Grande-Bretagne, ce qui poussa les archéologues de ce pays à s'intéresser à ce territoire. À partir de 1922, Leonard Woolley mena douze campagnes de fouilles à Ur, financées par le British Museum de Londres et l'University Museum de Philadelphie : il resta sur le site chaque année pendant plusieurs mois, avec une équipe d'encadrement d'environ cinq personnes, dont un épigraphiste. De fait, la moisson de textes a été impressionnante et pas moins de six assyriologues travaillèrent sur le chantier: Sidney Smith (Ire campagne, 1922-1923), Cyril John Gadd (II campagne, 19231924), Léon Legrain (III e et IVe campagnes, 1924-1925 et 1925-1926), Eric Burrows (Ve à VIII ${ }^{e}$ campagnes, de 1926-1927 à 1929-1930), Chauncey Winckworth (IX ${ }^{\mathrm{e}}$ campagne, 1930-1931) et enfin Cyrus Gordon (X $\mathrm{X}^{\mathrm{e}}$ campagne, 1931-1932).

En 1934, la mission de Woolley s'acheva; non que le site ait été épuisé, mais la nouvelle loi des antiquités alors promulguée mit fin au partage des objets découverts. Les grands musées occidentaux qui finançaient des fouilles en Irak arrêtèrent leur soutien en guise de protestation. Woolley se consacra alors à la publication de ses découvertes, qui avait déjà commencé. Il organisa deux séries parallèles, l'une consacrée à l'archéologie $(\mathrm{UE}=$ Ur Excavations) et l'autre à l'épigraphie $(\mathrm{UET}=U r$ Excavations Texts). Les très abondantes informations recueillies, portant sur plus de cinq millénaires, ont permis à Woolley de publier un rapport archéologique définitif en neuf volumes, mais il avait dû procéder à une sélection du matériel. Le projet « Ur online » (http://www.ur-online.org/), réalisé entre 2013 et 2016, place à la disposition de tous l'intégralité de la documentation conservée à Londres et à Philadelphie : photographies, fiches, carnets de fouilles, etc., sont directement consultables sur la Toile, avec de nombreux liens.

2. D. Charpin, Archives familiales et propriété privée en Babylonie ancienne. Étude des documents de "Tell Sifr », Genève/Paris, Droz/Champion, HEO 12, 1980. 
Les tablettes, pour la plupart en argile crue, ont été cuites sur le chantier, selon une technique courante à l'époque ; elles ont ensuite été envoyées au British Museum pour étude. Leur publication a été faite en neuf volumes selon une répartition typologique et chronologique, sans qu'aucune attention ait été portée à la provenance des textes ; il s'agit d'ouvrages traditionnels, comportant un catalogue, des index et des copies. C'est seulement après leur publication que les tablettes furent partagées entre les musées de Londres, Philadelphie et Bagdad; malheureusement, la répartition fut faite de façon purement mécanique, qui aujourd'hui handicape les recherches par lots d'archives. Par ailleurs, tout n'avait pas été publié au moment du partage, de sorte qu'il est resté de nombreux fragments inédits, en particulier pour l'époque d'Ur III et la période paléo-babylonienne, qui ont été publiés par la suite dans des livres ou des articles.

\section{Cours 2 - Histoire des fouilles et des publications (suite) ; I'histoire d'Ur, de la chute d'Ur III à l'abandon sous Samsu-iluna}

\section{1 avril 2018}

La première partie du cours a poursuivi l'historique des fouilles et des publications. L'étude des textes d'époque paléo-babylonienne a connu un nouvel élan à partir de 1976, lorsque fut enfin publié le rapport de fouilles que Woolley et Max Mallowan avaient achevé depuis des années (UE 7). Grâce au catalogue des objets (p. 214-254), il fut enfin possible de situer dans leur contexte archéologique les textes, qui avaient été publiés de manière typologique: documents d'archives (UET 5), exercices scolaires (UET 1, 7 et 8) ou «textes littéraires et religieux» (UET 6). Dès l'achèvement de ma thèse de troisième cycle en 1979, je me suis attelé à cette tâche, qui aboutit en 1984 à une thèse d'État dont est issu Le Clergé d'Ur, paru en $1986^{3}$.

On a ensuite retracé les principales découvertes effectuées lors des deux nouvelles campagnes de fouilles, à l'automne 2015 et au printemps 2017. Elisabeth Stone reprit l'exploration du quartier dit « $\mathrm{AH} »$, au sud-est du cœur de la ville, avec un triple objectif. Il s'agissait d'abord de parfaire notre connaissance des maisons dégagées par Woolley. La fouille a montré qu'il s'était souvent arrêté au premier niveau rencontré, sans rechercher les phases plus anciennes des demeures qu'il explorait ; c'est ainsi que de nombreuses tombes ont pu être découvertes. Le deuxième objectif était de retrouver les habitations de la fin du troisième millénaire dont les ruines devaient se trouver sous le niveau paléo-babylonien. Pour ce faire, deux sondages ont été effectués dans des secteurs déjà fouillés par Woolley : le premier (« Area 1 ») se situe au « $\mathrm{n}^{\mathrm{o}} 1$ Baker's square », le second («Area 2 ») dans « Niche Lane». Ce dernier a permis la découverte d'un lot de 18 textes de comptabilité de l'époque d'Akkad, une période jusqu'à présent fort mal représentée à Ur.

E. Stone souhaita enfin élargir la zone explorée par Woolley, ce qui fut fait en bordure du quartier AH, au nord-ouest («Area $3 »)$ et au sud («Area $4 »)$. L'idée était que la fouille de maisons paléo-babyloniennes avec les méthodes modernes de l'archéologie permettrait de progresser dans nos connaissances de ce quartier d'habitation, grâce notamment à l'étude des restes végétaux et animaux qui ne se pratiquait pas dans les années 1930, ainsi qu'à une étude plus précise des vestiges matériels communs auxquels

3. D. Charpin, Le Clergé d'Ur au siècle d'Hammurabi (XIXe-XVIII siècles av. J.-C.), Genève/ Paris, Librairie Droz, HEO 22, 1986. 
Woolley ne prêtait guère attention : son analyse de la céramique, par exemple, ne prenait en compte que les formes complètes et pas les tessons.

En 2017, la fouille de deux maisons dans les chantiers 3 et 4 du quartier AH, déjà entamée en 2015, a été poursuivie, tandis qu'un troisième chantier plus au sud était ouvert par une équipe allemande dirigée par Adelheid Otto, permettant la découverte d'une nouvelle maison ("Area 5 »). Le chantier 3 a révélé les archives du général Abisum, qui occupa cette demeure depuis la conquête par Hammu-rabi jusqu'à l'abandon de la ville à la fin de l'an 11 de Samsu-iluna. Au chantier 5 ont été découverts les vestiges d'une belle maison paléo-babylonienne. On y a découvert des tablettes et scellements mis au rebut qui ont permis d'établir que cette demeure avait été habitée jusqu'en 1835 av. J.-C. par l'intendant du temple de Ningal, nommé Sin-nada ; celui-ci se livrait aussi à son domicile à la formation d'apprentis-scribes, ce dont témoignent différents exercices. Le chantier 4 a également fourni des tablettes scolaires paléo-babyloniennes; pour finir, une couche contenant des tablettes de l'époque d'Ur III a été atteinte.

La seconde partie du cours a retracé l'histoire de la ville d'Ur à l'époque paléobabylonienne. Dans cette période de 260 ans, on peut distinguer quatre phases de longueur inégale. De 2004 à 1926 av. J.-C., après la chute de la troisième dynastie, Ur perdit son statut de capitale et fut remplacée dans ce rôle par Isin, où Išbi-Irra occupait déjà le trône. Dans le nom de sa 29e année (1991), il commémora la construction de trônes pour Nanna et Ningal, et dans le nom de sa $31^{\mathrm{e}}$ année, il se vante d'avoir «installé Ur fermement dans son emplacement»; toutefois, aucun texte n'a été conservé pour la vingtaine d'années qui sépare l'an 24 d'Ibbi-Sin d'Ur de l'an 5 du roi d'Isin Su-ilišu (2003 à 1982). Le contrôle d'Ur par Isin lui fut par la suite disputé par une nouvelle dynastie installée à Larsa : depuis l'an 7 du roi de Larsa Gungunum (1926) et pendant six décennies, la ville changea de mains plusieurs fois. À partir de l'avènement de Nur-Adad (1865) et durant le siècle qui suivit, Ur appartint au royaume de Larsa de manière stable. Enfin, en 1763, le royaume de Larsa - qui comprenait donc la ville d'Ur - fut annexé par Hammu-rabi de Babylone ; cette phase dura un quart de siècle, jusqu'en 1738, moment où la ville fut abandonnée pour plusieurs siècles.

\section{Cours 3 - La ville d'Ur : environnement et urbanisme}

2 mai 2018

L'étude de l'environnement naturel de la ville d'Ur et de son urbanisme est forcément multidisciplinaire. On a d'abord souligné l'apport considérable de la télédétection, depuis les vues aériennes de la Royal Air Force des années 1920 jusqu'aux images du drone employé sur le site par Emily Hammer en 2017, en passant par les photos satellitaires anciennes (Gambit KH-7 de l'US Air Force en 1966, Corona KH-4B du programme parallèle de la CIA 1968) ou plus récentes (Quickbird [2002, 2004, 2007], Ikonos-2 [2008] et WorldView-1 [2008]) ${ }^{4}$.

4. Voir le travail récent de G. Di GIACOMO et G. SCARDOZZI, « Multitemporal high-resolution satellite images for the study and monitoring of an ancient Mesopotamian city and its surrounding landscape: The case of Ur », International Journal of Geophysics, 2012 (Article ID 716296), DOI : 10.1155/2012/716296. 
Dans un premier temps, le cadre naturel dans lequel la ville d'Ur s'insérait a été décrit. On a rappelé que le cours de l'Euphrate s'est déplacé : au début du $\mathrm{II}^{\mathrm{e}}$ millénaire avant notre ère, il coulait à proximité de la ville, au sud, comme le montrent les traces d'un paléo-méandre révélées par un cliché de 1966. Un autre élément crucial est celui du tracé de la côte. La question est très complexe, car les facteurs sont multiples: affaissement de la plaine (compactage des alluvions, mouvements tectoniques), mais, inversement, alluvionnement du fait des fleuves, et, enfin, variation du niveau marin. Le point a été fait par Paul Sanlaville en 1989 : il y a eu un maximum de transgressions marines vers 3500 av. J.-C. La côte devait alors passer non loin d'Ur, et par les villes modernes de Nassiriya et Amara, donc à $200 \mathrm{~km} \mathrm{au}$ nord de la côte actuelle. Par la suite, les fleuves ont travaillé très efficacement et colmaté rapidement le fond de ce golfe, dont les profondeurs étaient faibles, sans doute en moins de deux millénaires. Depuis 2000 av. J.-C. environ, on observe une alternance de phases de recul et d'avancée du rivage, liées à la fois à de faibles oscillations du niveau marin et à des déplacements de l'embouchure des fleuves. Un élément très important de l'environnement dans la région est constitué par la zone des marais ; ce secteur était assez densément peuplé, car de nombreuses habitations en roseaux étaient installées sur des îles artificielles. La région a été volontairement asséchée par Saddam Hussein, luttant contre les déserteurs qui s'y étaient réfugiés; elle a été depuis heureusement remise en eau. Pendant la période d'assèchement, Abdulamir Al-Hamdani a pu prospecter cette zone, qu'il a étudiée dans sa thèse ${ }^{5}$.

La région autour des villes d'Ur et Eridu a fait l'objet d'une prospection d'Henry Wright en 1966, publiée seulement en 1981 ; le total des sites repérés dans cette zone s'élève à 192 - toutes périodes confondues. Certains sites ont été fouillés et, pour une partie d'entre eux, identifiés. Tel est le cas de Tell Abu Shahrein, à 12 km au sud-sud-ouest d'Ur, l'antique Eridu, ville du dieu Enki/Ea. Le site de Tell Obeid pourrait être à identifier avec Nutur ; celui de Tell Sakhariya avec Karzida/Gaeš. Il reste encore de nombreux toponymes qu'on ne sait où localiser précisément, comme Ki'abrig. Inversement, certains sites fouillés n'ont pas encore révélé leur nom, comme Tell Khaiber.

La dernière partie du cours a tenté de reconstituer le cadre urbain d'Ur. La ville haute s'étendait sur 60 hectares, mais la télédétection montre que la ville basse était beaucoup plus vaste que Woolley ne l'a indiqué : pas moins de 440 hectares. On a ensuite procédé à une sorte de « visite guidée » du site, en commençant par la zone sacrée du centre : l'Ekišnugal, sanctuaire du dieu-Lune (nommé Nanna en sumérien et Sin en akkadien) était dominé par la ziggourat. Il comportait aussi un grand entrepôt, le Ganun-mah, tandis que le Dubla-mah était une sorte de chapelle où les serments étaient prêtés. Non loin de là, le Gipar-ku était à la fois le temple de la déesse Ningal, parèdre de Nanna, et la résidence de la prêtresse-entum; les prêtresses décédées étaient enterrées dans un caveau souterrain, comme dans les maisons privées. Au sud-ouest de la zone sacrée, un quartier d'habitation a été fouillé (secteurs EH et EM), dans lequel Woolley a découvert plusieurs maisons habitées par des familles du clergé. Au sud de la ville, le quartier $\mathrm{AH}$ a également livré de nombreuses demeures. Woolley a aussi mis au jour les temples d'Enki et de

5. A. Al-Dafar [= Al-Hamdani], Shadow States: The Archaeology of Power in the Marshes of Southern Mesopotamia, New York, PhD Stony Brook University, 2015. 
Ningišzida. Il faut ajouter que les textes documentent des éléments importants qui n'ont pas été retrouvés par les fouilles jusqu'à présent, comme la muraille et les portes de la ville.

\section{Cours 4 - L'Ekišnugal, sanctuaire du dieu Nanna/Sin}

\section{6 mai 2018}

De nombreux temples existaient à Ur à l'époque paléo-babylonienne. Certains ont été retrouvés lors des fouilles, comme celui voué à Enki, ou encore le temple de Ningišzida; d'autres sont seulement attestés par les textes, comme ceux de Ningublaga, de Gula, etc. Le sanctuaire le plus important était celui de la divinité poliade d'Ur, le dieu-Lune, nommé Nanna ou Sin, qui avait pour parèdre la déesse Ningal ; comme tous les temples, il portait un nom cérémoniel, en l'occurrence celui d'Ekišnugal, « Temple (brillant comme) l'albâtre ».

Le dossier de la topographie de l'Ekišnugal est particulièrement compliqué, tant pour l'analyse des vestiges que pour celle des données textuelles. Le problème principal pour l'archéologie est que ce complexe, bâti pour l'essentiel au XXI ${ }^{\mathrm{e}}$ siècle av. n.è., a été constamment remanié jusqu'au $\mathrm{VI}^{\mathrm{e}}$ siècle : il n'est pas toujours aisé de démêler ce qui appartient à telle ou telle phase. D'autres difficultés sont dues aux informations lacunaires dans la documentation de la fouille, comme le manque de points topographiques dont l'altitude soit indiquée de façon fiable. Des progrès ont été faits par Martin Gruber, dans un article tout récent, dont certaines conclusions ne peuvent toutefois pas être retenues ${ }^{6}$. Ce que Woolley a nommé téménos correspond à l'espace enclos dans le mur d'époque néo-babylonienne ; mais à la période paléobabylonienne, cet espace était plus restreint. C'est à l'époque d'Ur III que les grandes lignes de l'organisation du sanctuaire ont été fixées. L'idée-force du projet architectural mis en place sous Ur-Nammu, le premier roi de la III ${ }^{\mathrm{e}}$ dynastie d'Ur, consistait à structurer l'espace par le moyen de plateformes et terrasses surperposées, ce qui avait un triple avantage : rendre le sanctuaire visible de loin, séparer le temple de la ville environnante, mais aussi délimiter des espaces spécifiques à l'intérieur du téménos. Deux charges étaient liées à l'existence de cet espace : les prébendes de portier $\left(\right.$ né- $\left.\mathrm{du}_{8}\right)$ et de balayeur (kisal-luh). Leurs titulaires étaient chargés de contrôler l'entrée du sanctuaire et de veiller à sa propreté. L'ensemble était dominé par la masse de la tour à étages : cette ziggourat avait un nom cérémoniel distinct de celui du temple proprement dit, en l'occurrence E-temen-nigur, «Temple, fondation revêtue de terreur». Il s'agissait d'une construction en briques crues avec un parement de briques cuites jointoyées au bitume, mesurant à la base $62,5 \mathrm{~m} \mathrm{x} 43 \mathrm{~m}$. Woolley n'a retrouvé que le premier étage, culminant à $11 \mathrm{~m}$ de hauteur, et l'amorce du second. Sa reconstitution s'appuie sur l'étude de la pente des escaliers : il estima qu'il n'y avait au total que trois étages. La cour au pied de la ziggourat semble désignée par le terme de kisal-mah ; c'est là que se trouvait la cella du temple, le papâhum. Le terme de kisal-sag-an-na semble avoir désigné ce que Woolley décrivit comme la « cour de Nanna », située dans la partie nord du complexe religieux.

6. M. GRUBER, « The topography of the temenos at Ur and its changes from the Third Dynasty to the Kassite Period », in K. KANIUTH, D. LAU et D. WiCKE (dir.), Übergangszeiten. Altorientalische Studien für Reinhard Dittmann anlässlich seines 65. Geburtstags, Münster, Zaphon, 2018, marru 1, p. 171-193. 
La deuxième partie du cours a été dévolue à l'analyse du culte. On a mis l'accent sur les soins donnés quotidiennement au dieu Nanna/Sin, autrement dit à sa statue, qui était lavée et ointe avant d'être vêtue. Les dieux devaient également être nourris, et leur alimentation préparée. Au pied de la ziggourat, les deux lieux essentiels étaient le four $\left(\mathrm{gir}_{4}\right)$ et la salle-à-manger $\left(\mathrm{unu}_{2}\right.$-gal). On a retrouvé des cylindres en cuivre portant une dédicace du roi Nur-Adad, commémorant la reconstruction du « grand four qui fournit constamment la nourriture au dieu Sin, qui produit le pain pour l'ensemble des dieux, soigneusement équipé sur la haute estrade de leur salle-à-

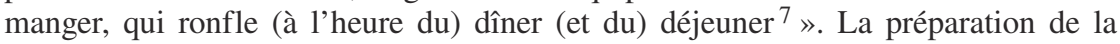
nourriture était en effet une activité rituelle accomplie par des purificateurs placés sous le patronage du dieu Kusu ; la charge de cuisinier, comme celle de brasseur, faisait partie des fonctions dans le temple qui, à l'époque paléo-babylonienne, pouvaient être négociées en tant que prébendes.

Il existait cependant d'autres types d'offrandes qu'alimentaires. Elles étaient notamment effectuées par toutes les personnes qui voulaient se propitier la divinité, et en premier lieu les rois. Ces derniers commémoraient parfois leurs offrandes dans des noms d'années ; dans d'autres cas, on a retrouvé la dédicace rédigée à cette occasion. Les souverains offraient des statues de divinités, des trônes ou des emblèmes, ou encore des statues d'eux-mêmes en posture d'orant. Nous possédons en outre des témoignages écrits qui documentent des visites royales dans l'Ekišnugal. Le premier groupe a trait au roi de Larsa Rim-Sin. Les textes concernant sa visite ont été découverts dans la maison de purificateurs-abriqqum au $\mathrm{n}^{\circ} 7$ Quiet Street du quartier EM. Il s'agit de trois hymnes, d'une cinquantaine de lignes chacun, qui semblent avoir été lus à différents moments du déplacement du cortège dans le complexe sacré. Ils se caractérisent par la formule finale : « Ô Rim-Sin, mon roi ! » et n'ont pas de duplicat. Dans les archives du général babylonien Abisum découvertes au printemps 2017, deux tablettes enregistrent une dépense de poissons pour le «repas du roi » au début de l'année 5 du règne de Samsu-iluna : cela nous montre que le souverain babylonien était alors venu jusqu'à Ur, fait jusqu'à présent ignoré. Son voyage avait manifestement pour but d'offrir en personne un trône au dieu Nanna/Sin, don jugé assez important par le roi pour être commémoré dans la formule qui donna son nom à l'année 5.

\section{Cours 5 - La place du sanctuaire de Nanna dans la vie économique et juridique d'Ur}

\section{3 mai 2018}

Le principal bâtiment lié à la vie économique du sanctuaire de Nanna était le Ganun-mah. Il est connu à la fois par l'archéologie et par un grand nombre de sources écrites : des inscriptions commémoratives (noms d'années et inscriptions royales), mais aussi des documents d'archives. L'édifice se situe à l'est du téménos, dans l'angle entre la cour de Nanna et la terrasse de la ziggourat. Il se présentait sous la forme d'un carré de 57 mètres de côté, entouré par un mur épais de 2,70 mètres. Le plan se caractérise par la présence de pièces parallèles très allongées avec ouverture sur l'un des petits côtés, typique des espaces de stockage dans l'architecture mésopotamienne.

7. D.R. Frayne, Old Babylonian Period (2003-1595 BC), Toronto, University of Toronto Press, RIME 4, 1990, p. 140-142 n 3. 
Le Ganun-mah avait une double fonction. C'était d'abord un lieu où étaient déposés l'argent du temple, ainsi que des objets précieux ; il servait aussi au stockage des denrées périssables destinées aux offrandes alimentaires faites à Nanna et aux autres divinités. Cette dualité ressort des inscriptions royales, mais aussi des documents d'archives : il s'agit de tablettes périmées mises au rebut sous le sol de certaines pièces. Certaines ont été retrouvées sur place ; d'autres ont été découvertes dans deux maisons du quartier AH. Le lot YOS 5 nos 1-110 appartient également à la comptabilité du Ganun-mah, mais sa provenance archéologique est inconnue puisque ces tablettes proviennent de fouilles irrégulières effectuées avant la Première Guerre mondiale. Beaucoup de ces documents sont indiqués comme ayant été scellés par les « intendants » (̌́atammû). Ce terme indique une fonction, mais ce n'est pas un titre : on trouve en effet souvent parmi ces sceaux celui du šandabakkum, i.e. du responsable économique du sanctuaire.

C'est dans la caisse du Ganun-mah qu'était versé l'argent résultant de la vente de certains biens du temple, qu'il s'agisse de transactions exceptionnelles comme l'aliénation de terrains ou de ventes plus courantes comme celle de poissons. Beaucoup d'objets précieux étaient offerts au dieu Nanna à titre d' « offrandes » plus ou moins volontaires. Une grande partie de la comptabilité du Ganun-mah a trait aux troupeaux de bovins. Le contexte écologique permet de comprendre qu'il s'agissait avant tout de buffles qui vivaient dans les marais, selon des modalités qui existent encore de nos jours. Le temple de Nanna à Ur n'était pas seulement propriétaire d'importants troupeaux de bovins: les activités de laiterie qu'il abritait étaient conçues comme intrinsèquement liées à la nature du dieu, comme l'indiquent certains hymnes ${ }^{8}$. Nanna n'était pas seulement compris comme protecteur des bovins, il était lui-même l'un d'eux ; son animal attribut était le taureau, le croissant de lune étant vu comme les cornes de l'animal. D'autres divinités proches de Nanna avaient également un lien étroit avec les troupeaux et l'élaboration de produits laitiers.

Les pièces de comptabilité retrouvées mises au rebut au $\mathrm{n}^{\mathrm{o}} 1$ Broad Street concernent également les roseaux. Ceux-ci servaient à de nombreux usages : la construction de bâtiments, la fabrication d'objets (nattes, récipients, etc.), mais aussi l'alimentation des animaux : il y avait donc un lien avec les troupeaux de bovins. Par ailleurs, certains des individus mentionnés dans ce lot d'archives étaient en relation avec les pêcheurs, ce qui n'a rien d'étonnant. Le centre de leurs activités semble avoir été la ville de Ku'ara, dans les environs d'Eridu.

Les denrées conservées dans le Ganun-mah étaient l'huile, le beurre, le fromage, les dattes, le miel et divers aromates. En revanche, les céréales et le pain étaient livrés par le «grenier de Nanna», le karûm (sumérien GUR $_{7}$ ), connu par un lot d'une vingtaine de tablettes découvertes dans le « Mausoleum site », c'est-à-dire à l'extérieur du téménos paléo-babylonien.

À Ur, le Dubla-mah était le lieu où les serments exigés par les juges devaient être prêtés, comme le montrent plusieurs textes de procès. Une inscription d'Amar-Sin décrit cet édifice comme l'endroit du jugement de Nanna et le compare à un «filet auquel aucun ennemi ne peut échapper ». Une catégorie particulière de prébendiers était rattachée à ce bâtiment (les gudu 4 dub-lá-mah), chargés d'organiser le rituel qui entourait chaque prestation de serment.

8. D. Charpin, La Vie méconnue des temples mésopotamiens, Paris, Les Belles Lettres/ Collège de France, coll. « Docet omnia », 2017, p. 180. 


\section{Cours 6 - Le Gipar-ku : princesses et religion}

30 mai 2018

Au sud-ouest du sanctuaire de Nanna se trouvait un bâtiment décrit par plusieurs inscriptions comme Gipar-ku, soit « Gipar pur». Il était lié à une fonction religieuse remplie par une personne portant le titre sumérien de «en » : à Ur, il s'agissait d'une femme, qu'on désignera ici par son titre akkadien d'entum. Une inscription de l'une de ces prêtresses, nommée Enanedu, contient cette définition de son rôle : «L'être qui a le grand rôle de bâtir en un lieu pur le Gipar pour en assurer la prêtrise (nam-en), c'est moi !»

Le bâtiment en question se situe dans le téménos, au sud-ouest de la ziggourat ; il date pour l'essentiel de l'époque d'Ur III, des travaux importants ayant été effectués à l'époque paléo-babylonienne. Le Gipar-ku était presque carré, mesurant $79 \mathrm{~m}$ x 76,50 m, et orienté par les angles. L'intérieur était coupé par un couloir transversal, d'où la distinction faite par Woolley entre deux ensembles : le bloc sudest (C) était le temple de Ningal, le bloc nord-ouest $(\mathrm{A}+\mathrm{B})$ formait la résidence de l'entum. On trouve à la limite entre ces deux ensembles des réserves (B1 à B8) et une cuisine (C32-34), qui servaient à la fois à la déesse et à la prêtresse.

Chaque prêtresse-entum était fille du roi qui dominait Ur au moment où elle était choisie par divination, puis installée dans sa fonction : les deux événements furent souvent commémorés par un nom d'année. Elles portaient toutes un nom sumérien en rapport avec leur charge, comme en-me-gal-an-na « entum (apte) aux grands rites de An », en-šà-ki-ág-d nanna « entum bien-aimée de Nanna » ou en-an-e-du 7 « entum qui convient à An ». Les activités cultuelles des entum sont malheureusement fort mal documentées ; on en sait davantage sur leur gestion du domaine qu'elles devaient gérer. À leur mort, elles étaient placées dans un caveau funéraire situé sous le bâtiment; chaque entum devait procurer des offrandes funéraires à celles qui l'avaient précédée. Il est aujourd'hui possible d'améliorer sur certains points la prosopographie des prêtresses-entum qui avait été élaborée par Johannes Renger en 1967. On a pu corriger une erreur ancienne de Gadd : Enšakiag-Nanna était morte lorsque Enanedu occupa la charge d'entum, et la notion de « retraitée », dans ce cas comme dans tous les autres en Mésopotamie, est à oublier ${ }^{9}$. Il n'y avait donc qu'une seule entum vivante à la fois.

La partie sud-est du Gipar-ku était occupée par un sanctuaire, manifestement celui de la déesse Ningal. Le lieu le plus important était désigné comme agrun (akk. kummum), soit la « chambre »; un texte mythologique indique que c'est l'endroit où Ningal avait mis au monde son fils, le dieu Utu/Šamaš. Ce temple était géré par un administrateur-šangûm et des intendants-abarakkum; différents prébendiers lui étaient rattachés. La fin du cours a été consacrée aux découvertes faites par A. Otto et son équipe de l'université de Munich en mars 2017, dans une grande demeure située à plusieurs centaines de mètres au sud du quartier AH. Les textes ont été découverts sous le dernier niveau d'occupation : ils ont manifestement été mis au rebut en 1835 av. J.-C., lorsque Sin-nada quitta cette demeure, donc un siècle avant que la ville d'Ur ait été abandonnée sous le roi de Babylone Samsu-iluna. La légende de son sceau comporte après son nom quatre lignes, dont les trois premières indiquent qu'il était « 2 fils de Igi-

9. D. CHARPIN, «En marge d'Archibab, 25 : une offrande à Ur d'Etellum, ministre du roi de Larsa Gungunum », NABU, 2017-2, p. 75-77 no 42. 
anakezu, ${ }^{3}$ scribe, ${ }^{4}$ intendant (ugula é) du temple de Ningal ». Une des lettres découvertes dans la maison était adressée à une femme nommée Nuțtuptum, très vraisemblablement l'épouse de Sin-nada : après avoir dressé la liste des objets qu'il lui faisait parvenir, il ajoutait qu'il rentrerait dix jours plus tard, ce qui confirme que la maison où la tablette a été trouvée était sa résidence.

\section{Cours 7 - Le clergé et son habitat}

6 juin 2018

Le cas de la prêtresse-entum était particulier: sorte de doublure humaine de Ningal, l'épouse du dieu Nanna, elle habitait le bâtiment qui abritait aussi le temple même de la déesse. Mais il est clair que telle n'était pas la règle générale. Nous avons d'abord étudié le quartier proche du sanctuaire de Nanna, à savoir les sites EH et EM, pour tenter de déterminer quelle proportion de desservants du temple y résidait et quel était le statut du sol dans cette zone.

Le quartier EH fut d'abord fouillé par Taylor en 1854, les tablettes qu'il découvrit ayant été transportées au British Museum. Dans les décennies qui suivirent, des fouilles non contrôlées eurent manifestement lieu au même endroit, les tablettes exhumées ayant été dispersées entre de nombreuses collections. C'est lors de sa quatrième campagne, en 1925-1926, que Woolley reprit la fouille de cette zone. Malheureusement, le secteur était si mal conservé qu'il ne put pas vraiment dégager de plan cohérent, à l'exception d'un temple voué à Nimintabba. La dévotion privée à cette déesse, à l'époque paléo-babylonienne, est assez bien documentée, mais uniquement à Ur, la plupart des témoignages ayant été trouvés dans ce secteur. Il s'agit donc d'un exemple très intéressant de l'enracinement d'un culte spécifique dans un quartier limité. Nimintabba était une déesse gardienne du temple de Nanna ; un temple lui était dévolu en propre à proximité du grand sanctuaire. On voit à quel point la vie des dieux était calquée sur celle des hommes : les portiers du temple, titulaires de prébendes, vivaient eux aussi dans des maisons aux alentours du sanctuaire. Ce quartier EH se poursuivait plus à l'ouest, dans une zone qui fut appelée EM par Woolley ; il y fit un essai à la fin de sa IVe campagne, à proximité de l'endroit déjà sondé avec succès par Hall en 1919, et les résultats encourageants qu'il obtint le décidèrent à poursuivre. Cela fut fait en 1926-1927, lors de la $\mathrm{V}^{\mathrm{e}}$ campagne, durant laquelle Woolley dégagea une surface environ $2800 \mathrm{~m}^{2}$, mettant au jour trois axes de circulation et une quinzaine de maisons. Les archives découvertes montrent qu'ont vécu dans ce quartier plusieurs générations de familles appartenant au clergé de l'Ekišnugal. C'est ainsi qu' au « n 7 Quiet Street » ont successivement habité l'économe (šandabakkum) du temple de Nanna, Ur-Nanna, puis le prêtre-abriqqum Ku-Ningal et ses fils. Ils avaient notamment pour voisin l'administrateur ( ̌́atammum) Ela, et un cultivateur du domaine de la déesse Ningal, Sin-ereš. Dans la maison « $\mathrm{n}^{\circ} 5$ Quiet Street » vécut le kišibgallum Šamaš-nașir. En principe, le terrain était la propriété des familles qui habitaient ces demeures. Un seul exemple montre le temple de Nanna vendre une parcelle dans ce quartier : il s'agit sans doute d'une maison en déshérence. Il faut ajouter que ce quartier n'était pas exclusivement habité par le clergé du temple voisin et inversement que certains membres du personnel du sanctuaire pouvaient habiter ailleurs dans la ville, comme on l'a vu avec Sin-nada.

La seconde partie du cours a mis l'accent sur un groupe particulier au sein du clergé de l'Ekišnugal, celui des personnes qui se définissaient par rapport au dieu Enki- 
d'Eridu. Le site d'Eridu, très important pour les périodes protohistoriques et encore au $\mathrm{III}^{\mathrm{e}}$ millénaire, avait connu un déclin marqué au début du $\mathrm{II}^{\mathrm{e}}$ millénaire ; une inscription de Nur-Adad parlait bien de la reconstruction du sanctuaire, mais jusqu'à présent on n'a retrouvé que fort peu d'éléments datant de cette époque. Or, il se trouve qu'au sein du clergé demeurant à Ur, certains individus se définissaient par rapport au dieu Enki, mais en mentionnant qu'il s'agissait d' «Enki d'Eridu». Il faut d'emblée souligner l'étrangeté de cette précision : d'habitude, un dieu était nommé seul, même s'il était la divinité poliade d'une ville. On ne désigne par exemple jamais le dieu-Lune comme « Nanna d'Ur » ou « Sin d'Ur ». On a donc recensé les attestations de ces titres et des personnes qui les portaient. On a ensuite étudié en détail le cas de deux familles de purificateurs-abriqqum particulièrement bien connues et dont les membres se revendiquaient comme rattachés à «Enki d'Eridu ». La première famille est celle de Nanna-addani et de son fils E-igidubi-isilim. La fouille de 2017 a fourni une nouvelle attestation de ce dernier (le texte a été étudié en séminaire). La deuxième famille est encore mieux connue : Ku-Ningal et ses cinq fils occupèrent dans le quartier EM la maison « $\mathrm{n}^{\circ} 7$ Quiet Street». On a retracé leur histoire et exposé la tradition liée aux dieux d'Eridu dont ils étaient les dépositaires. Dans l'onomastique d'Ur, le nom Eriduliwwir («Puisse Eridu briller !») manifeste l'attachement de ce groupe à une ville qu'il avait dû quitter, mais dont le souvenir se transmettait de génération en génération.

\section{Cours 8 - Le quartier AH et la vie économique d'Ur}

\section{3 juin 2018}

Le quartier dit «AH», situé au sud du sanctuaire de Nanna, constitue aujourd'hui encore le plus vaste ensemble urbain jamais fouillé en Mésopotamie, sur une surface d'environ $8000 \mathrm{~m}^{2}$, ayant permis le dégagement d'une cinquantaine de maisons. D'un point de vue social, il semble avoir été plus mélangé que les quartiers EH et EM.

La fouille de ce quartier par Woolley a été effectuée en une seule campagne (ce qui parait aujourd' hui incroyable), sa IX ${ }^{\mathrm{e}}$, du $1^{\mathrm{er}}$ novembre 1930 au 20 mars 1931 . Sous les restes mal conservés du niveau néo-babylonien, Woolley découvrit des maisons plus anciennes, remontant à la seconde moitié du $\mathrm{II}^{\mathrm{e}}$ millénaire (époque kassite). C'est seulement en dessous qu'apparut le premier niveau complètement conservé, datant de la première moitié du II ${ }^{\mathrm{e}}$ millénaire, qui fit l'objet d'un dégagement en extension. Lorsque E. Stone reprit la fouille du site en 2015, son but était double : essayer de retrouver, sous le niveau paléo-babylonien, la couche de l'époque d'Ur III qu'on suppose avoir été présente. L'idée était de comparer les deux périodes, de façon à voir si les différences très fortes que l'on constate actuellement ne sont pas dues à une comparaison faussée par le hasard des fouilles, qui ont touché plutôt des quartiers domestiques pour la période paléo-babylonienne et plutôt des bâtiments publics pour l'époque d'Ur III. Le deuxième objectif était de fouiller de nouvelles maisons en bordure de la zone dégagée par Woolley, de façon à bénéficier de toutes les avancées techniques de l'archéologie moderne: au début des années 1930, il n'y avait pas d'analyses des macro-restes végétaux, ni des ossements humains ou animaux découverts. Les observations plus fines devaient aussi permettre de mieux interpréter les données relevées par Woolley. Deux zones furent choisies dans le quartier AH pour reprendre la fouille. Dans la maison « $\mathrm{n}^{\circ} 1$ Baker's square» («Area $\left.1 »\right)$, la surprise vint du fait que la fouille de Woolley avait été très incomplète. Il fallut notamment dégager le caveau funéraire qu'il avait repéré mais pas fouillé ; comme tous les autres, 
il avait été pillé lorsque le quartier fut abandonné en 1738 av. J.-C. D'autres inhumations, non construites cette fois, furent découvertes sous le sol des pièces 6 et 7 , ce qui ralentit le travail et ne permit d'atteindre le niveau inférieur que sur une surface très réduite. Dans le chantier rouvert dans « Niche Lane » (« Area 2 »), un sondage en profondeur dans la rue permit de s'enfoncer. Il n'y eut pas de niveau Ur III important comme on s'y attendait, mais la principale surprise est venue de la découverte d'un lot de 18 textes de comptabilité de l'époque d'Akkad. La principale extension se situe au nord-ouest du quartier AH (« Area $3 »)$. Elle permit de découvrir une grande maison, conforme aux normes architecturales de l'époque paléo-babylonienne; les archives découvertes - 45 tablettes - montrent que le dernier occupant du bâtiment fut un général babylonien nommé Abisum, sur lequel on est revenu lors du cours $\mathrm{n}^{\mathrm{0}} 10$.

La première caractéristique du plan du quartier $\mathrm{AH}$ est son caractère labyrinthique. Les rues n'y sont pas larges et ne suivent aucun tracé préconçu. Il s'agit plutôt de l'espace laissé libre entre les bâtiments ; on relève même l'existence de nombreuses impasses. La technique de construction des maisons consistait à monter les assises inférieures des murs en briques cuites, surmontées par un mur de briques crues; les pièces étaient le plus souvent dallées de carreaux également cuits. La restitution des espaces en trois dimensions pose des problèmes proprement architecturaux, sur lesquels l'ethno-archéologie peut également apporter un éclairage. A-t-on affaire au milieu des maisons à une cour, comme Woolley se l'imaginait pour la maison « $\mathrm{n}^{\mathrm{o}} 3$ Gay Street», qu'il a prise comme modèle, ou s'agit-il de ce que Jean Margueron appelle de façon plus neutre un «espace central», qu'il suppose le plus souvent couvert ? Les escaliers qui ont été retrouvés menaient-ils à une terrasse, ou sont-ils la preuve de l'existence d'un étage ? Autant de problèmes qu'il est difficile de trancher. On a également abordé la question des prétendues « chapelles » et des tombes.

Le quartier AH est célèbre d'un point de vue historiographique pour la découverte de la maison d'Ea-nașir ( « $\mathrm{n}^{\mathrm{o}} 1$ Old Street »), un marchand qui faisait du commerce maritime avec Tilmun, soit l'île de Bahrein. C'est pourquoi la seconde moitié du cours a été consacrée au commerce à longue distance pratiqué depuis Ur, en utilisant les données du quartier $\mathrm{AH}$ et en les complétant par les informations venues d'autres locus déjà étudiés, en particulier le Ganun-mah. L'importance du commerce maritime se voit par le fait que l'une des offrandes les plus courantes faites au temple de Ningal était constituée par des maquettes de bateaux. Selon A. Leo Oppenheim, suivi par Wilhelmus François Leemans, les dons (A.RU.A) et les dîmes (ZAG.10) étaient des sortes d'ex-voto offerts par les marchands lorsqu'ils revenaient sains et saufs de leur expédition jusqu'à Tilmun ; Marc Van De Mieroop a proposé que la « dîme » n'ait pas eu de caractère volontaire, mais ait été une taxe de $10 \%$ prélevée par les temples. La maison d'Ea-nașir a livré des lettres qui documentent le commerce de façon très vivante : des investisseurs confiaient d'importantes sommes d'argent à des marchands qui faisaient le déplacement jusqu'à Tilmun, d'où ils rapportaient du cuivre en quantité. Tilmun était donc une sorte de plaque tournante dans un commerce qui allait au-delà, le cuivre provenant de la péninsule d'Oman.

\section{Cours 9 - La formation des scribes et le problème de la littéracie}

20 juin 2018

Pendant longtemps, le rôle du clergé mésopotamien dans l'éducation a été l'objet de débats. La ville d'Ur a fourni des données très riches, permettant de voir quel rôle jouait 
l'écrit dans la formation des apprentis et dans la transmission des traditions. Nous avons successivement étudié ce que nous apprend la maison « $\mathrm{n}^{0} 7$ Quiet Street»; puis le problème posé par le « $\mathrm{n}^{\circ} 1$ Broad Street », que Woolley a considéré comme une école ; et enfin les informations nouvelles qui viennent des fouilles de 2017.

Le père Burrows, qui était l'épigraphiste de la mission à Ur en 1926-1927 quand Woolley fouilla le quartier EM, écrivit avec hésitation à propos de la maison « $\mathrm{n}^{\circ} 7$ Quiet Street»: «perhaps a scribal school». Nous avons étudié lors du cours $\mathrm{n}^{\circ} 7$ l'histoire des purificateurs qui vécurent dans cette maison et leurs traditions particulières qui mettaient en avant le dieu Enki d'Eridu et les divinités de son entourage; nous sommes revenus sur les tablettes scolaires qu'on y a découvertes. On n'y trouve pas trace des phases élémentaires de l'apprentissage, mais des copies de séries lexicales et de textes grammaticaux qui servaient à l'enseignement de la langue sumérienne. Un exercice plus difficile consistait à copier des lettres en sumérien : une tablette comprend le texte de six lettres, dont deux ne sont pas attestées ailleurs. On a aussi quelques tablettes mathématiques. La copie d'inscriptions historiques était également un travail d'apprentissage courant : on en possède trois pour l'époque d'Akkad, trois pour l'époque d'Ur III, deux de rois d'Isin et trois de rois de Larsa ; certaines comportent des erreurs grossières, d'autres sont écrites avec une main malhabile. Outre ces textes «classiques », la maison « $\mathrm{n}^{\mathrm{O}} 7$ Quiet Street» contenait également des textes plus atypiques. Si la copie de listes de noms propres était un exercice fréquent, celle qui a été retrouvée est très particulière : il s'agit d'une liste de noms sumériens de prêtres qui portaient des noms à la louange du temple qu'ils desservaient et dont la tablette donne la traduction en akkadien. UET 6117 a ceci d'unique que les noms qu' on y trouve sont ceux de prêtres collègues des purificateurs habitant la maison où l'exercice a été retrouvé : on a sans doute ici le reflet d'un enseignement, le maître expliquant (en akkadien) à un élève le sens des noms sumériens portés par des clercs de sa famille ou de son entourage, que l'élève côtoyait sans doute quotidiennement. On a aussi retrouvé des hymnes qui n'ont de parallèle nulle part ailleurs, et qui sont clairement liés aux traditions et activités des purificateurs-abriqqum voués au dieu Enki d'Eridu, comme on l'a déjà vu lors du cours $n^{0} 7$. Ce que l'étude du « $n^{0} 7$ Quiet Street » a apporté de très nouveau lorsqu'elle a été publiée en 1986, c'est une vision différente de l'apprentissage de l'écriture de celle qui prévalait alors. La synthèse qui faisait alors autorité, due à A Sjöberg, reposait en grande partie sur les textes littéraires décrivant les activités dans l'école (sumérien é-dub-ba): on voyait celle-ci comme une institution indépendante, installée dans des locaux réservés à l'enseignement. Le cas $\mathrm{du} \ll \mathrm{n}^{\mathrm{o}} 7$ Quiet Street $»$ montre que le caractère familial de la transmission du savoir écrit, considéré par les assyriologues comme typique de la seconde moitié du $\mathrm{II}^{\mathrm{e}}$ millénaire et du $\mathrm{I}^{\mathrm{er}}$ millénaire, remontait en fait à l'époque paléo-babylonienne.

L'endroit qui a déjà été du temps de Woolley considéré comme «l'école » par excellence à Ur se trouve dans le quartier $\mathrm{AH}$, au « $\mathrm{n}^{\mathrm{O}} 1$ Broad Street». Les découvertes épigraphiques dans cette maison furent très abondantes, mais l'épigraphiste d'alors, Chauncey Winckworth, n'a pas été à la hauteur de la situation, de sorte qu'une bonne partie des tablettes ont perdu leur numéro d'inventaire et n'ont pu par la suite être identifiées ; on a trouvé une majorité d'exercices scolaires, mais aussi des documents d'archives. En 1986, il avait été proposé que pratiquement toutes les tablettes de cette maison n'y aient pas été retrouvées in situ, mais qu'elles y aient été mises au rebut. En 2000, Paolo Brusasco a tenté de revenir à l'interprétation initiale de Woolley, qui avait eu l'impression de découvrir les 
tablettes «tombées de leurs étagères ». La clé réside dans l'interprétation des archives. Or, celles-ci n'ont aucune cohérence, ni thématique ni chronologique : on a bien l'impression de tablettes mises au rebut - dès lors, pourquoi n'en serait-il pas de même pour les tablettes scolaires ? Ceci ne fait cependant que repousser la question : d'où venaient ces tablettes scolaires ? Il est bien difficile de le dire. Mais mieux vaut avoir une vision sainement critique plutôt que d'en revenir à des rapports de fouilles manifestement obsolètes.

Heureusement, on n'en reste pas à ces controverses. En effet, les fouilles de 2017 ont apporté une contribution inespérée au dossier de l'apprentissage de l'écriture. Dans le «secteur 4 » ont été retrouvés plusieurs exercices scolaires qui relèvent d'un enseignement d'initiation et portent, comme c'est souvent le cas, des signes de recyclage. Par ailleurs, la maison de Sin-nada, l'intendant du temple de Ningal, présentée lors du cours $\mathrm{n}^{\circ} 6$, a également livré des exercices scolaires, notamment des tablettes lenticulaires. Mais l'apprentissage assuré par Sin-nada à son domicile ne se limitait pas aux rudiments, car un manuscrit de la Lamentation sur Sumer et Ur a également été découvert dans cette maison.

\section{Cours 10 - La domination babylonienne et sa fin}

\section{0 juin 2018}

Pour la plupart des auteurs, c'est pendant la quarantaine d'années qui va de l'avènement de Warad-Sin au milieu du règne de Rim-Sin (de 1834 à 1793) qu'Ur connut son apogée à l'époque paléo-babylonienne, avant que Hammu-rabi n'annexe le royaume de Larsa et que de ce fait Ur se retrouve intégrée au royaume de Babylone. Mais on aurait tort de considérer le quart de siècle de domination par Hammu-rabi (1763-1750), puis Samsu-iluna (1749-1738), comme une période de déclin. On relève pendant ces vingt-cinq ans un contraste entre une histoire politico-militaire chahutée et, par ailleurs, de nombreux éléments de continuité révélés par les archives.

La période commence par la conquête du royaume de Larsa par Hammu-rabi : aucune information précise n'a trait à la chute d'Ur. Dans le prologue du Code de Hammu-rabi, la place faite à Ur, à son dieu Nanna et à son sanctuaire, l'Ekišnugal, juste après Babylone, Marduk et l'Esagil, est remarquable ; elle contraste avec le peu de traces laissées sur place par le conquérant babylonien. Au bout de treize ans, Hammu-rabi mourut et son fils Samsu-iluna lui succéda. Il n'a laissé aucune inscription à Ur, mais on sait désormais qu'il ne négligea pas la ville : un texte découvert en 2017 dans la maison d'Abisum montre que le roi vint en personne à Ur offrir le trône au dieu Nanna mentionné dans le nom de l'année 5. On constate qu'il n'existe à Ur aucun texte entre le milieu du onzième mois de l'an 8 de Samsu-iluna et le milieu du huitième mois de l'an 10, donc pendant un an et neuf mois : c'est durant cette période que les habitants d'Ur reconnurent le rebelle Rim-Sin II ; contrairement à ce qui a été souvent écrit, celui-ci ne prit cependant pas le titre de « roi d'Ur ». Samsu-iluna réussit à reprendre la totalité du Sud durant sa dixième année de règne. La muraille d'Ur fut détruite, de même que celle d'Uruk où avait régné l'usurpateur Rim-Anum.

En dépit de cette histoire politique agitée, l'historien relève de nombreux éléments de continuité : la plupart du temps, ce sont les mêmes familles qui continuèrent à exercer leurs responsabilités, depuis le règne de Rim-Sin I jusqu'à la fin de l'occupation paléo-babylonienne de la ville. On a mis l'accent sur la reconstitution 
en cours de quelques archives. Certaines proviennent des fouilles régulières, notamment celles du chef-maçon Ibbi-Ilabrat (trouvées dans une tombe du site EH), du responsable des magasins du temple (kišibgallum) Šamaš-nașir (EM, « ${ }^{0} 5$ Quiet Street »), d'Ili-ippalsam ( $\mathrm{AH}, \ll \mathrm{n}^{\circ} 1$ Store Street »). On a aussi des archives issues des fouilles clandestines anciennes.

Le cours s'est terminé par la question de la fin de l'occupation d'Ur. Une approche historiographique a permis de relever un grand nombre d'erreurs. La plus grave a été commise par Woolley lui-même : à cause du nom d'année mentionnant les murailles d'Ur, le fouilleur a été obsédé par le souci de trouver des traces de la destruction par Samsu-iluna. Mais ce nom d'année est celui de l'an 11, qui commémore un événement de l'année antérieure. Et l'on trouve dans les bâtiments des textes datés de l'an 11 de Samsu-iluna : c'est la preuve que malgré la destruction de la muraille qui eut lieu l'année d'avant, la vie avait repris. L'année 11 de Samsu-iluna est attestée à Ur par 14 tablettes, datées du 1/i au 30/xii, et elles ne montrent rien d'anormal. Après, c'est le silence... Dans deux cas, des tablettes ont été trouvées dans des tombes, où elles ont dû glisser lors de l'ouverture de celles-ci : il est donc clair qu'un pillage suivit immédiatement l'abandon des maisons par leurs habitants, qui y laissèrent leurs archives, au moins en partie. La fouille de 2017, avec les archives d'Abisum trouvées autour de la tombe et à l'intérieur de celle-ci, a confirmé cette interprétation: les maisons ont été pillées immédiatement après que leurs habitants les ont quittées, juste après la fin de l'année 11 de Samsu-iluna. Un texte - exceptionnel par sa date - prouve qu'un groupe de prêtres resta pour surveiller les sanctuaires (UET 5 868, édition dans HEO 22, p. 209). Ce document, daté du mois viii de l'année 12 de Samsu-iluna, montre trois personnages chargés de veiller au bon accomplissement des tâches de portier et de balayeur dans le temple de Ningal pour les six prochains mois. La garde du temple de la déesse Ningal fut donc alors réorganisée : il s'agissait manifestement d'éviter que les pillards qui rôdaient ne s'emparent des trésors qui s'y trouvaient encore... Et c'est finalement ce qui se produisit : le temple de Ningal a été trouvé très ruiné par Woolley. Les multiples traces de destruction qu'il a repérées - et attribuées à Samsu-iluna - sont dues en fait à des pillards. Ce n'est pas un hasard si elles sont particulièrement nettes dans le secteur des tombes : c'est là que les voleurs espéraient trouver les objets les plus précieux. Nous savons maintenant ce qui se passa ensuite : la région passa sous la coupe d'une dynastie nouvelle, celle du «Pays de la Mer». Mais la vie urbaine ne reprit qu'après la reconquête des rois kassites de Babylone, dans le courant du $\mathrm{XIV}^{\mathrm{e}}$ siècle av. J.-C.

\section{SÉMINAIRES - TEXTES RELATIFS À LA VILLE D'UR}

La première séance a porté sur les deux seuls contrats de vente immobilière par le temple de Nanna connus à Ur. On sait que ce sanctuaire était un grand propriétaire foncier, mais ces deux contrats documentent la vente de petites parcelles dont on a pu préciser le contexte urbain : il s'agit manifestement de terrains en déshérence qui sont vendus à des voisins par le temple, ce dernier jouant un rôle ailleurs dévolu aux municipalités.

Le reste du séminaire a été consacré à l'étude des archives découvertes à Ur dans une maison du quartier $\mathrm{AH}$, lors des fouilles qui ont eu lieu au printemps 2017. J'ai pu copier les quelque 45 tablettes et fragments exhumés dans ce bâtiment, dont je 
prépare l'édition : il s'agit des archives d'un certain Abisum, qui constituent jusqu'à présent la première trace directe de la présence de Babyloniens dans la ville d'Ur, suite à l'annexion du royaume de Larsa par Hammu-rabi lors de sa trentième année. Cet Abisum était en effet un général babylonien (portant le titre de UGULA MAR. TU); il n'était pas encore connu et ses archives documentent ses activités sous le règne de Hammu-rabi (années 36 et 43), puis sous le fils et successeur de celui-ci, Samsu-iluna, jusqu'à l'an 11, période après laquelle la ville fut abandonnée.

On a commencé par un texte particulièrement intéressant: il s'agit du remboursement par Abisum d'une dette contractée par un de ses subordonnés envers un individu nommé E-igidubi-isilim. Or, celui-ci était déjà connu par les archives d'une famille habitant le secteur EM : E-igidubi-isilim y figurait comme témoin dans un contrat daté du dernier roi de Larsa, en 1769. Son nom apparaît aussi dans un texte scolaire consistant en une liste de noms propres (UET 6 117) : le nom d'E-igidubiisilim est traduit en akkadien par « temple dont la vue procure la santé ». On aurait pu croire que le créancier E-igidubi-isilim dans la tablette d'Abisum était un homonyme du prêtre attesté 20 à 30 ans plus tôt. Mais le reçu de l'argent remboursé par Abisum a été scellé, et le sceau comporte la même légende que sur des tablettes collationnées en 1987 : il s'agit bien du même E-igidubi-isilim, attesté sur une période de plusieurs décennies. Cela permet de voir que ces purificateurs n'avaient manifestement pas une place marginale dans la ville d'Ur, et purent la conserver indépendamment des aléas politiques que connut leur cité ; on constate qu'ils ne répugnaient pas à entretenir des relations économiques avec les occupants babyloniens.

On a ensuite étudié les responsabilités d'Abisum en tant que général. Il était assisté par deux « colonels » (UGULA GIDRI). On le voit notamment affecter un soldat à la zone des marais ( $a-n a$ hal-și-im a-na A.AB.BA). Cette région toute proche d'Ur relevait de son commandement et cela explique pourquoi plusieurs textes comptabilisant des livraisons de poissons ont été retrouvés dans sa maison. Deux des tablettes des archives d'Abisum enregistrent une dépense de poissons pour le « repas du roi » au début de l'année 5 du règne de Samsu-iluna, ce qui montre que le souverain babylonien était alors venu jusqu'à Ur.

Les archives d'Abisum révèlent par ailleurs les activités économiques habituelles de ce genre de notables : il louait des champs qu'il cultivait, embauchait du personnel pour quelques mois, et effectuait de nombreux prêts, autant de contrats qui nous sont parvenus en plus ou moins bon état. L'absence de lettres doit être soulignée : on peut penser qu'elles se trouvaient dans une pièce de la maison qui n'a pas encore été fouillée, ce qui donne bon espoir pour la campagne prévue au printemps 2019.

\section{COURS À L'EXTÉRIEUR - FAIRE REVIVRE LA CIVILISATION MÉSOPOTAMIENNE : DE L'ARGILE À INTERNET}

Cours donné dans le cadre d'une convention entre le Collège de France et l'Académie royale de Belgique, le 17 janvier 2018 au Collège Belgique Namur ${ }^{10}$.

Après une introduction rappelant brièvement les origines de la discipline nommée « assyriologie » par Ernest Renan, par analogie avec l' « égyptologie », la conférence a commencé par décrire les principales caractéristiques de la documentation écrite

10. Voir https://www.youtube.com/watch ?time_continue $=8 \& \mathrm{v}=\mathrm{LLRG} \_$XZpBAE. 
que la civilisation mésopotamienne a livrée et qui conditionnent le travail de l'assyriologue. Dans un deuxième temps, un panorama des différents types de sources a montré la très grande diversité des approches possibles de cette civilisation, malgré son éloignement dans le temps. Pour finir, on a présenté les moyens actuels de la recherche et de la diffusion de l'information à l'âge des humanités numériques. J'ai mis l'accent sur l'expérience acquise sur des chantiers de fouille en Syrie (Mari) et en Irak (Larsa et, depuis 2015, Ur) et sur les travaux de mon équipe, qui est très impliquée dans la création d'outils informatiques à tous les stades du travail assyriologique.

À l'invitation de l'École biblique et archéologique française de Jérusalem, j'ai donné, entre le 12 et le 16 mars 2018 , deux cours et un séminaire.

\section{Mari et la Bible : un bilan provisoire}

En introduction, l'historique des recherches sur le tell Hariri, l'antique Mari, a été brièvement retracé ; puis un état des lieux de la publication des milliers de tablettes découvertes dans le palais a été effectué, avec près de 9000 textes actuellement édités, soit environ les deux tiers de ce qui est réellement utilisable. Enfin, quelques éléments historiographiques de la thématique «Mari et la Bible» ont été indiqués.

Dans un premier temps, nous nous sommes attachés au cadre géographique et institutionnel, avec un accent particulier mis sur le problème du tribalisme et de la vie nomade. Nous avons ainsi examiné le statut de villes telles que Hazor, décrite dans Josué 11, 10 comme ayant été une capitale importante dans le passé, et qui, de fait, joue un rôle très marquant dans les archives de Mari. À Mari, les gouverneurs portaient le titre amorrite de šâpițum; cela permet de mieux comprendre le terme de šophet, dont la traduction traditionnelle par « juge » ne rend pas compte de manière satisfaisante. Les textes de Mari documentent la vie des nomades amorrites au XVIII ${ }^{\mathrm{e}}$ siècle avec une quantité considérable de détails, comme la pratique de cairns funéraires qui rappellent celui qui fut érigé sur la tombe d'Absalon ; des données nouvelles permettent de mieux connaître la transhumance qui s'effectuait chaque année depuis le Liban jusqu'au bassin du Habur. La royauté de Mari, du temps de son dernier roi, Zimri-Lim, a pu être qualifiée de «royauté tribale » et peut certainement aider à comprendre la monarchie des débuts de l'histoire d'Israël.

Dans un deuxième temps, nous nous sommes penchés sur les phénomènes religieux. Le dossier du prophétisme a été le premier qui ait retenu l'attention. Pendant longtemps, on a considéré que l'existence de prophètes était une caractéristique de la Bible. On sait depuis quelques décennies que ce n'est pas vrai : il y a eu des prophètes au Proche-Orient depuis au moins le XVIII ${ }^{\mathrm{e}}$ siècle av. J.-C. Une autre erreur a eu la vie plus dure : l'idée d'une division du Proche-Orient en deux. L'Ouest aurait été le domaine des prophètes, l'Est celui des devins, Mari se situant à l'intersection des deux ensembles. En réalité, on s'aperçoit que l'hépatoscopie était pratiquée à l'Ouest : on le sait grâce à Mari pour Alep et Qatna, les fouilles de Hazor l'ont montré aussi. Par ailleurs, on connaît des prophéties dans la partie orientale du Proche-Orient, notamment dans le royaume d'Ešnunna et à Babylone même. Une bibliographie considérable a été consacrée à la comparaison entre le prophétisme tel qu'il apparaît dans les archives de Mari et celui qui est illustré par les écrits bibliques. Nous devons commencer par rappeler que, s'il n'est pas question d'une filiation directe de Mari à la Bible, on ne se trouve pas dans le cas 
d'un comparatisme "exotique » comme lorsque l'on compare le prophétisme de Mari avec le chamanisme: il y a bel et bien un continuum culturel dans les civilisations du Proche-Orient antique. Et si les prophéties néo-assyriennes sont contemporaines de celles qu'on trouve dans la Bible, les deux ensembles sont également héritiers des pratiques du début du $\mathrm{II}^{\mathrm{e}}$ millénaire. Comme dans tout sain comparatisme, il faut à la fois souligner les ressemblances et les différences. Des ressemblances ont été relevées quant aux procédures, aux formulations et enfin au contenu même des prophéties. S'il ne faut jamais oublier que des siècles séparent les deux corpus, de nature profondément différente, il convient aussi de souligner que bien des efforts pour mettre en évidence l'«originalité de la prophétie dans la Bible » se sont peu à peu révélés vains, à mesure que le corpus de Mari grossissait. Il existe cependant des différences. L'une d'elles réside dans le lien très fort entre le prophétisme attesté par les archives de Mari et le caractère anthropomorphique des représentations divines. L'absence de relations entre «maître » et "disciples » est un des éléments expliquant la différence essentielle, qui tient à la façon dont les prophéties nous sont parvenues. À Mari, la mise par écrit des prophéties est uniquement affaire de circonstances : il s'agit de faire connaître au roi une prophétie qui lui est destinée ou qui le concerne. Aucun phénomène de réécriture a posteriori n'affecte ces textes, et c'est ce qui doit faire tout leur prix aux yeux des biblistes soucieux de comparatisme.

L'interdiction de représenter Dieu est un des points forts du Décalogue. À Mari, la religion la mieux connue est celle de type mésopotamien, où les dieux sont représentés sous forme de statues. Mais on a peu à peu découvert que Mari attestait aussi une religiosité aniconique. Cet aspect a d'abord été révélé par la fouille du temple de « Nini-zaza », dans les années 1950. Dès 1985, Jean-Marie Durand a fait connaître des textes qui montraient que les bétyles avaient occupé une place importante dans la religion à Mari au XVIII ${ }^{\mathrm{e}}$ siècle.

Pour finir, on a étudié la façon dont les alliances étaient conclues. La comparaison entre les données de la Bible et celles de l'époque paléo-babylonienne a porté sur plusieurs éléments: le rite de l'immolation d'un ânon, l'étymologie du mot hébraïque pour «alliance » (běrît) et enfin l'obligation de sincérité. On a ajouté un élément nouveau, qui ne semble pas encore être entré dans la littérature du côté des biblistes : le rôle du sang dans la conclusion des alliances.

\section{Les remises de dettes, de Sumer à nos jours ${ }^{11}$}

En Mésopotamie, il existait deux termes que l'on peut traduire par «justice». Le premier, kittum, dérive de kânum « être stable » : c'est le Droit en tant que garant de l'ordre établi. Le second, mîšarum, dérive du verbe ěsêrum «aller droit». On comparait le roi à un berger qui «fait aller droit» son troupeau : il devait éviter que les bêtes s'égarent et les conduire vers pâturages et points d'eau. Ainsi entendue, la Justice-mî̌sarum traduit la sollicitude du roi guidant son peuple. À ce titre, les souverains promulguaient des édits dont certains aspects se retrouvent dans la Bible et ont eu des prolongements jusqu'à nos jours.

11. Voir http://www.ebaf.edu/2018/04/video-les-remises-de-dettes-de-sumer-a-nos-jours-viala-bible/. 
Les édits de mî̌sarum des rois mésopotamiens contenaient des prescriptions de natures diverses. Il s'agissait d'abord de mesures internes à l'administration, remettant aux débiteurs leurs arriérés, tant en ce qui concerne la production que la commercialisation. Le roi intervenait également dans les relations entre ses sujets en effectuant la remise des emprunts de nécessité contractés par les plus pauvres pour tenir jusqu'à la prochaine récolte. Cette mesure était accompagnée de deux autres complémentaires, désignées par le terme andurârum : le retour des personnes à leur statut d'origine, qui permettait aux esclaves pour dettes d'être libérés, et l'annulation des ventes de biens effectuées pour rembourser des dettes. Des mesures similaires se retrouvent dans la Bible dans le cadre de «l'année sabbatique» (šemitțah; Lévitique 25 et Deutéronome 15): tous les sept ans, les Hébreux placés en gage doivent être libérés par les créanciers et l'on doit remettre leurs dettes aux débiteurs insolvables. Ces mesures sont complétées dans Lévitique 25 par ce qu'on désigne comme «le Jubilé » ou «l'année jubilaire », qui a lieu tous les cinquante ans (soit après sept cycles de sept ans) et qui est annoncé au son de la trompe (yobel) : « l'affranchissement» ( $\left.d^{e} r o ̂ r\right)$ consistait en la mise en jachère des terres, au retour des biens aliénés à leur ancien propriétaire et à la libération des esclaves pour dettes.

Le mot $\underline{D}^{e} \underline{R} \hat{o} R$ est construit sur la même racine que l'akkadien an $\underline{D} u \underline{R} \hat{a} \underline{R} u m$ (DRR) : cela conduit à mener une comparaison systématique entre les données mésopotamiennes et celles de la Bible, et à poser le problème d'une éventuelle filiation. Certaines mesures prescrites dans la Bible sont très différentes de celles des édits des rois babyloniens, mais d'autres très semblables, à commencer par la remise des dettes. En Mésopotamie, au départ, un édit était lié à l'avènement du roi ; par la suite, les souverains durent en proclamer à plusieurs reprises au cours de leur règne, mais jamais selon un rythme fixe, au contraire de ce que l'on trouve dans la Bible. Grâce aux nombreux documents d'archives à notre disposition, nous pouvons constater que les édits de mî̌sarum des rois babyloniens étaient réellement appliqués. Il en va tout autrement des mesures prescrites dans la Bible; le seul indice d'application - ou plutôt de non application - que nous ayons figure dans le livre de Jérémie (34 8-16).

Malgré les différences, on voit donc qu'il y a des points communs importants entre les édits des rois mésopotamiens et les prescriptions bibliques : la question qui se pose alors est de savoir s'il existe une filiation historique. Pendant longtemps, la parenté entre les pratiques paléo-babyloniennes et les prescriptions bibliques a posé un problème qui semblait insoluble : celui des siècles qui les séparent. Or, des études récentes ont montré que l'annulation des dettes s'est poursuivie en Mésopotamie au-delà de 1600. Le fait que l'andurârum soit maintenant bien documentée à l'époque néo-assyrienne, non seulement dans les inscriptions royales, mais aussi dans les textes de la pratique, permet de comprendre comment la transmission a été possible.

La pratique de la remise des dettes n'est pas limitée au Proche-Orient des $\mathrm{II}^{\mathrm{e}}$ et $\mathrm{I}^{\mathrm{er}}$ millénaires avant notre ère. Elle a eu un héritage qui conduit jusqu'à nous : les lois d'amnistie suivant l'élection des présidents de la Ve République (jusqu'en 2007) étaient un héritage régalien qui remontait à l'Ancien Régime et, de là, jusqu'à la Bible. Cette étude des édits de rémission des rois babyloniens rejoint des préoccupations très contemporaines : le mouvement pour l'abolition de la dette des pays en voie de développement, qui a connu un point culminant en 2000, s'appuyait explicitement sur les prescriptions bibliques, elles-mêmes héritières de la pratique mésopotamienne. 


\section{Bilan et perspective des recherches sur l'époque paléo-babylonienne}

On a commencé par un bilan de la situation sur le terrain. Celle-ci a été marquée par les pillages de nombreux sites. En Irak, ceux-ci ont commencé dès la période de l'embargo postérieur à la Guerre du Golfe de 1991 et se sont accrus après l'invasion américaine de 2003. En Syrie, la situation s'est tragiquement détériorée à partir de 2011. Des éléments positifs sont toutefois à noter : la recherche archéologique au Kurdistan, qui s'était interrompue dès les années 1960, a repris avec vigueur, mais n'a pas encore livré de textes d'époque paléo-babylonienne. Dans le sud de l'Irak, des missions de plus en plus nombreuses reprennent le travail.

La publication des textes se poursuit. Le bilan des dix dernières années s'établit à 3400 textes d'archives paléo-babyloniennes, qui proviennent des acquisitions des musées, mais aussi des fouilles, anciennes ou récentes, en Irak et en Syrie.

La recherche est également marquée par la constitution de nouveaux outils : il s'agit avant tout de bases de données disponibles sur la toile. Une présentation du site www.archibab.fr a été effectuée.

Quelques thématiques sur lesquelles de grands progrès ont été faits cette dernière décennie ont ensuite été développées : la culture matérielle (avec les trois volumes des Matériaux pour le Dictionnaire de babylonien de Paris dans la série des $\mathrm{ARM}^{12}$ ), la géographie historique (avec le projet « HIGEOMES »), la comptabilité (avec le projet «Comptabab»), le rapport de la langue et de l'écriture, le rôle des femmes, ou les alliances.

On a terminé en déplorant la lenteur avec laquelle les données et les idées nouvelles se diffusent : par exemple, on peut lire encore qu'il n'existe pas de traités écrits à l'époque paléo-babylonienne, alors que, depuis 1986, une dizaine a été publiée. Pour la têbibtum à Mari, certains auteurs renvoient encore à un article pionnier de 1950, définissant le terme comme un rite de purification alors qu'il s'agit du recensement... Les recensions et particulièrement les chroniques bibliographiques publiées chaque année dans la Revue d'Assyriologie essaient d'aider les assyriologues et les autres spécialistes à se frayer un chemin dans une actualité riche qui témoigne que ces recherches sont un secteur scientifique en pleine expansion.

\section{CONFÉRENCIERS INVITÉS}

Elizabeth Stone, professeur au département d'anthropologie de l'université Stony Brook (New York, États-Unis), a donné quatre conférences ${ }^{13}$ : le 16 mai 2018 («Understanding Mesopotamian Settlement Systems »), le 23 mai 2018 (« Survey and Soundings at Mashkan-shapir»), le 30 mai 2018 («Survey and Soundings at Tell Sakhariyah») et 6 juin 2018 (« New Excavations at Ur»).

Paul Zimansky, professeur au département d'histoire de l'université Stony Brook (New York, États-Unis), a donné une conférence ${ }^{14}$ le 28 mai 2018 ("City and Settlements in Urartu »).

12. Collection «Archives royales de Mari », $\mathrm{n}^{\text {os }} 30,31,32$, Paris, CNRS Éditions.

13. Disponibles en ligne : https://www.college-de-france.fr/site/dominique-charpin/ guestlecturer-2017-2018_1.htm.

14. Disponible en ligne : https://www.college-de-france.fr/site/dominique-charpin/ guestlecturer-2017-2018.htm. 


\section{RECHERCHE}

Le professeur et son équipe (en particulier Nele Ziegler, Antoine Jacquet, AnneIsabelle Langlois, Baptiste Fiette, Vérène Chalendar, Marine Béranger et Francesca Nebiolo, avec l'aide à distance de collègues étrangers, en particulier Ilya Arkhipov) ont continué à alimenter la base de données ARCHIBAB consacrée aux documents d'archives d'époque paléo-babylonienne. Il s'agit d'abord de suivre l'actualité : les 3400 textes nouveaux publiés ces dix dernières années, depuis le début du projet en 2008 jusqu'à la fin 2017, ont désormais tous été traités, souvent avec des progrès par rapport à l'édition publiée. Le travail rétrospectif a également continué parallèlement. Le site internet (www.archibab.fr) a été enrichi par l'inclusion d'un nouvel onglet relatif à la géographie historique, donnant accès au travail du projet franco-allemand « HIGEOMES » dirigé par N. Ziegler pour l'ANR. Par ailleurs, la série ARCHIBAB a été enrichie d'un nouveau volume : A.-I. Langlois, Archibab 2. Les archives de la princesse Iltani découvertes à Tell al-Rimah (XVIII siècle av. J.-C.) et l'histoire du royaume de Karana/Qațtara, Antony, SEPOA, coll. « Mémoires de NABU », $\mathrm{n}^{\circ} 18$, 2017. Un bilan du travail des dix premières années du projet ARCHIBAB a été préparé dans l'été 2018 et paraîtra dans le numéro 112 de la Revue d'Assyriologie.

Le projet «Digibarchi 15 », financé par l'université PSL depuis le 15 octobre 2015 jusqu'au 31 mars 2018, a permis de numériser toute la photothèque des archives royales de Mari (35 000 clichés argentiques) et de traiter les photos numériques prises depuis 1999. On a poursuivi la mise en ligne sur la base ARCHIBAB des photos des tablettes déjà publiées, la plupart de ces clichés n'ayant jamais été reproduits.

Le projet concernant la ville d'Ur dans le premier quart du $\mathrm{II}^{\mathrm{e}}$ millénaire retenu par l'ANR, nommé « EcritUr ${ }^{16} »$, a commencé au 1 ${ }^{\text {er }}$ octobre 2017. Une table ronde a été organisée par moi-même et Antoine Jacquet à la fondation Hugot les 17 et 18 mai 2018, réunissant tous les participants directs au projet, ainsi que des collègues étrangers s'intéressant à Ur dans les musées (J. Taylor du British Museum) ou sur le terrain (E. Stone et P. Zimansky de l'université Stony Broke, New York, ainsi que A. Otto, de la LMU de Munich ${ }^{17}$ ).

\section{PuBLiCATIONS}

CHARPIN D., « Le "mur des Amorrites" à Sumer à la fin du III ${ }^{e}$ millénaire av. J.-C. : le premier exemple de mur anti-migrants? », in P. BOUCHERON (dir.), Migrations, réfugiés, exil, Paris, Odile Jacob/Collège de France, 2017, p. 61-81.

ChARPIN D., « Chroniques bibliographiques 20. Pour une diplomatique des documents paléobabyloniens », Revue d'assyriologie et d'archéologie orientale, vol. 111, 2017, p. 155-178, DOI : $10.3917 /$ assy.111.0155.

ChARPIN D., «Dons ou ventes de terres par les rois à l'époque paléo-babylonienne », in G. Neumann, S. Paulus et K. Kleber (dir.), Grenzüberschreitungen. Studien zur Kulturgeschichte des Alten Orients. Festschrift für Hans Neumann anlässlich seines 65. Geburtstages am 9. Mai 2018, Münster, Zaphon, coll. « Dubsar », vol. 5, 2018, p. 99-142.

15. Cf. http://digitorient.com/?page_id=2693.

16. Cf. http://digitorient.com/programmes-de-recherche/3-projets-de-lequipe-3-mondesmesopotamiens/ ecritur-projet-anr-2017-2020/.

17. Cf. http://digitorient.com/blog/2018/05/10/premiere-table-ronde-ecritur-17-et-18-mai-2018/. 
CHARPIN D., «Schriftkultur in Babylonien: Plädoyer für eine Diplomatik der Keilschrifturkunden », in B. SCHNITZLEIN et E. CANCIK-KIRSCHBAuM (dir.), Keilschriftartefakte. Untersuchungen zur Materialität von Keilschriftdokumenten, Gladbeck, PeWe-Verlag, coll. « Beiträge zum Vorderen Orient », vol. 26, 2018, p. 145-160.

CHARPIN D., «Les "rois archéologues" en Mésopotamie : entre l'authentique et le faux », in H. GABER, N. GRIMAL et O. PERDU (dir.), Imitations, copies et faux dans les domaines pharaonique et de l'Orient ancien. Actes du colloque Collège de France - Académie des Inscriptions et Belles-Lettres, Paris, 14-15 janvier 2016, Paris, AIBL-Soleb, coll. «Études d'égyptologie », vol. 16, 2017, p. 176-197.

CHARPIN D., «Babylone. Tant d'énigmes encore à résoudre », Le Monde de la Bible, n 226, 2018, p. 62-65.

CHARPIN D., «Les bibliothèques en Mésopotamie: des fonds de manuscrits privés aux bibliothèques royales », in Y. LEHMANN (dir.), Savoir/Pouvoir. Les bibliothèques, de l'Antiquité à la modernité, Turnhout, Brepols, coll. «Recherches sur les rhétoriques religieuses », vol. 29, 2018, p. 65-79.

CHARPIN D., «67. En marge d'ARCHIBAB 26: deux devins et l'abi ERIN 2 Utul-Ištar à Sippar-Amnanum en Ammi-șaduqa 15 », NABU, vol. 2017/3, 2017, p. 124-125.

CHARPIN D., «11. En marge d'EcritUr, 1: un temple funéraire pour la famille royale de Larsa ?», $N A B U$, vol. 2018/1, 2018, p. 14-16.

CHARPIN D., « 12. En marge d'EcritUr, 2 : retour au no 7 Quiet Street », $N A B U$, vol. 2018/1, 2018, p. 16.

CHARPIN D., «13. En marge d'Archibab, 27 : les légendes de sceaux des UGULA DAM. GÀR », NABU, vol. 2018/1, 2018, p. 17.

CHARPIN D., «14. En marge d'Archibab, 28 : une offrande au dieu Panumundu à Larsa », $N A B U$, vol. 2018/1, 2018, p. 17-19.

Charpin D., «Civilisation mésopotamienne », Annuaire du Collège de France 2015-2016. Résumé des cours et travaux, vol. 117, 2018, p. 229-242, DOI : 10.4000/annuaire-cdf.12850 ; en ligne : https://journals.openedition.org/annuaire-cdf/12850.

CHARPIN D., How to Be an Assyriologist? Inaugural Lecture delivered on Thursday 2 October 2014, trad. de L. LIBBRECHT, Paris, Collège de France, coll. « Leçons inaugurales », 2017, DOI : 10.4000/books.cdf.4922, https://books.openedition.org/cdf/4922 [traduction anglaise de : Comment peut-on être assyriologue ?].

Ainsi que quatre recensions dans la Revue d'assyriologie et d'archéologie orientale, $\mathrm{n}^{\mathrm{o}} 111$, 2017.

FIETTE B., «68. Note sur les toponymes du Sud mésopotamien, 1 : Maškan-šar et l'alliance entre Rim-Sin de Larsa et l'Elam », $N A B U$, vol. 2017/3, 2017, p. 125-127.

FIETTE B., «69. Note sur les toponymes du Sud mésopotamien, 2: Kamum », NABU, vol. 2017/3, 2017, p. 127-128.

FIETTE B., «70. Note sur les toponymes du Sud mésopotamien, 3: Kar-Šamaš », NABU, vol. $2017 / 3,2017$, p. 128 .

FiETTE B., « 15. Note sur les toponymes du Sud mésopotamien, 4 : Al-marat-šarrim », $N A B U$, vol. 2018/1, 2018, p. 19-21.

FIETTE B., «16. Note sur les toponymes du Sud mésopotamien, 5 : Kar-Nabium », NABU, vol. 2018/1, 2018, p. 21-22.

FIETTE B., «Le domaine royal de Hammu-rabi de Babylone », Histoire et sociétés rurales, no 49, 2018, p. 9-53.

JACQuet A., Recension de J. LAUINGer, Following the Man of Yamhad. Settlement and Territory at Old Babylonian Alalah (Leyde/Boston, Brill, 2015), RA, n 111, 2017, p. 181-185. 